\title{
Rapid Quantitation of Aortic Valve Flow Using Spiral Fourier Velocity Encoded MRI
}

\author{
Joao L. A. Carvalho ${ }^{1}$ and Krishna S. Nayak ${ }^{2}$ \\ ${ }^{1}$ Universidade de Brasília \\ ${ }^{2}$ University of Southern California \\ ${ }^{1}$ Brazil \\ ${ }^{2} U S A$
}

\section{Introduction}

Aortic stenosis consists in a narrowing or incomplete opening of the aortic valve. This typically alters the blood flow, causing turbulent and/or complex flow jets. Such jets display peak velocities considerably higher than those of normal flow, and a much broader range of flow velocities. Another form of aortic valve disease is aortic insufficiency, or regurgitation. This condition occurs when the aortic valve fails to close completely. This is also known as "leaky valve", as flow jets in the reverse direction are observed when no flow should occur. The visualization and quantitation of cardiovascular blood flow is an important component of the assessment of aortic valve disease. For example, peak velocity measurements in flow jets are used to estimate pressure drop, which is an indicator of the hemodynamic load of a stenosis (Tsai et al., 1999).

\subsection{Doppler ultrasound}

The current non-invasive gold standard for flow quantitation is Doppler ultrasound. The ultrasound equipment is relatively inexpensive, small, and portable. Measurements are typically obtained in real-time, with excellent temporal resolution. The most popular techniques for ultrasound flow assessment are color Doppler and spectral Doppler.

Evaluation by ultrasound is impossible when there is air, bone, or surgical scar in the ultrasound path. Examination by ultrasound in obese patients is difficult, as the overlying adipose tissue (fat) scatters the sound waves. Doppler flow measurements may be inaccurate when the ultrasound beam cannot be properly aligned with the vessel axis, requiring measured velocities to be "angle-corrected" by the operator. Peak-velocity overestimation on the order of $18-40 \%$ have been reported in the literature (Hoskins, 1996; Winkler \& Wu, 1995), usually due to spectral broadening at large insonation angles, and to Doppler gain settings.

\subsection{Magnetic resonance imaging}

Magnetic resonance imaging (MRI) is potentially the most appropriate technique for addressing all aspects of cardiovascular disease examination, which includes assessing myocardial function and perfusion, as well as visualizing and measuring blood flow. MRI overcomes the acoustical window limitations of ultrasound, potentially allowing flow measurements to be obtained along any direction, and for any vessel in the cardiovascular 
system. Magnetic resonance (MR) measurements are also less operator-dependent than those of Doppler ultrasound, and the true direction of flow can generally be precisely measured. The main MR techniques for measuring flow are phase contrast and Fourier velocity encoding. These techniques are introduced below, and will be discussed in further detail in section 2 .

\subsubsection{Phase contrast}

Phase contrast (O'Donnell, 1985) is a technique in which a bipolar gradient (see section 2) aligned with the axis of flow is used to obtain a velocity measurement associated with each pixel (or "voxel") of the image. In practice, two acquisitions are used, and the first moment of the bipolar gradient is varied between measurements. The velocity estimate is obtained from the phase difference between the images obtained in each acquisition.

Phase contrast can be combined with dynamic (cine) MRI (Glover \& Pelc, 1988), in which short acquisitions and some form of cardiac synchronization are used to produce images throughout the cardiac cycle. The combined technique (cine phase contrast) can depict motion and flow throughout the cardiac cycle (Nayler et al., 1986). Alternatively, phase contrast can be combined with real-time MRI (Holsinger et al., 1990; Riederer et al., 1988), in which the encodings are applied sequentially, periodically, and continuously. In real-time MRI, images are formed by sliding a window along the acquired data and reconstructing an image for each position of the window. The display of real-time phase contrast data is typically implemented as color overlay of flow information (phase difference) over the anatomical (magnitude) image, which is called real-time color flow (Nayak et al., 2000; Riederer et al., 1991).

In phase contrast, data inconsistency, partial volume effects, and intravoxel phase dispersion can lead to peak velocity underestimation (Clarke et al., 1996; Tang et al., 1993). Partial voluming is particularly problematic when flow is highly localized and/or turbulent. When a large voxel size is adopted to measure the flow rate, not only may moving spins and stationary spins coexist in a voxel, but also the velocity distribution of spins within a voxel may spread over a wide range of velocities. This results in signal loss, distortion and erroneous velocity estimates. As a result, phase contrast imaging can not provide accurate peak velocity measurements in turbulent and/or complex flow jets. Such jets are commonly observed in narrowed vessels, and in valves presenting stenotis and/or regurgitation.

\subsubsection{Fourier velocity encoding}

The limitations mentioned above can be overcome using Fourier velocity encoding (FVE) (Moran, 1982). FVE can be considered the MR equivalent to spectral Doppler. In this technique, the full spectrum of velocities within each voxel is measured by phase-encoding the velocity information in Fourier domain. Therefore, FVE is robust to partial voluming, and flow measurements from low spatial resolution images are still accurate (Tsai et al., 1999). FVE shows satisfactory agreement with Doppler ultrasound (Mohiaddin et al., 1997). However, it is typically not used clinically, because the acquisition time required by this technique is in principle considerably longer than that of phase contrast.

Different approaches to accelerating FVE have been proposed. One example is the use of two-dimensional cylindrical excitation to restrict the field-of-view to a beam that can be imaged without phase encoding (Dumoulin et al., 1991). This approach makes it possible to perform spatial encoding and velocity encoding simultaneously, and in a single pulse repetition time (TR) (DiCarlo et al., 2005; Hu et al., 1993; Irarrazabal et al., 1993; Macgowan et al., 2005). This allows FVE measurements to be obtained in real-time. However, real-time FVE has problems related to the precise placement of the imaging beam, especially when the 
region of interest (e.g., mitral valve) moves during the cardiac cycle. Other problems include the large voxel size and low temporal resolution.

FVE has also been accelerated by simply neglecting spatial encoding along one of the spatial dimensions (Feinberg et al., 1985; Hennig et al., 1988), or by acquiring velocity images with no spatial encoding other than slice selection (Galea et al., 2002). In these techniques, the velocity measurement is a projection of all signal along a line or a plane in 3D space, respectively. As a consequence, both methods have dynamic range issues, as the signal of flowing blood has to be distinguished from all the background signal from static tissue observed along the projection. Furthermore, these approaches are unable to resolve different sources of flow that may co-exist in the projected line or plane.

\subsection{Chapter outline}

This chapter introduces spiral FVE, a novel method for MR flow quantitation that addresses the limitations discussed above. The proposed method provides fully-localized time-velocity distribution measurements, in a single acquisition, that is one short breath-hold long (approximately 10 seconds). Spiral FVE uses conventional slice-selective excitation (Bernstein et al., 2004; Nishimura, 2010), which excites (selects) a thin slice of the body to be imaged. The two-dimensional plane defined by this slice is imaged using spiral acquisitions (Ahn et al., 1986; Meyer et al., 1992), which encode both spatial dimensions simultaneously. Therefore, no spatial encoding is neglected, and measurements are fully localized in 3D space. 2D-resolved spatial encoding allows for easy localization of the region of interest, and the ability to resolve multiple sources of through-plane flow in the imaged field-of-view, without requiring static tissue suppression. Scan-plane prescription is performed using classic protocols, which is considerably less laborious than the beam-placing process used in real-time FVE.

Without acceleration, spiral FVE presents some limitations: (1) insufficient velocity field-of-view (the maximum range of velocities allowed without aliasing); (2) low in-plane spatial resolution, which limits the ability of spatially localizing the flow; (3) long readouts, which causes spatial blurring at $3 \mathrm{~T}$, due to off-resonance effects (Noll, Meyer, Pauly, Nishimura \& Macovski, 1991); and (4) moderate temporal resolution, which may blur certain features of the flow waveform. We address these limitations using the following acceleration techniques: variable-density spirals (Tsai \& Nishimura, 2000), partial Fourier reconstruction (Noll, Nishimura \& Macovski, 1991), and temporal acceleration (Madore et al., 1999; Tsao, 2002). By combining these techniques, we achieve a total 18-fold acceleration in spiral FVE.

\section{MR flow imaging}

\subsection{Basic principles of MRI}

MRI is a modality uniquely capable of imaging all aspects of heart disease, and is a potential "one-stop shop" for cardiovascular health assessment. MRI can generate cross-sectional images in any plane (including oblique planes), and can also measure blood flow. The image acquisition is based on using strong magnetic fields and non-ionizing radiation in the radio frequency range, which are harmless to the patient.

The main component of a MRI scanner is a strong magnetic field, called the $B_{0}$ field. This magnetic field is always on, even when the scanner is not being used. Typically, MR is used to image hydrogen nuclei, because of its abundance in the human body. Spinning charged particles (or "spins"), such as hydrogen nuclei, act like a tiny bar magnet, presenting a very small magnetic field, emanating from the south pole to the north pole. In normal conditions, 
each nucleus points to a random direction, resulting in a null net magnetization. However, in the presence of an external magnetic field (such as the $B_{0}$ field), they will line up with that field. However, they will not all line up in the same direction. Approximately half will point north, and half will point south. Slightly more than half of these spins (about one in a million) will point north, creating a small net magnetization $M_{0}$, which is strong enough to be detected. The net magnetization is proportional to the strength of the $B_{0}$ field, so MRI scanners with stronger magnetic fields (e.g., 3 Tesla) provide higher signal-to-noise ratio (SNR).

Another important component of the scanner are the gradient coils. There are typically three gradient coils $\left(G_{x}, G_{y}\right.$, and $\left.G_{z}\right)$, that produce an intentional perturbation in the $B_{0}$ field when turned on ("played"). This perturbation varies linearly along each spatial direction $(x, y$ and $z$ ), such that no perturbation is perceived at the iso-center of the magnet when these gradients are used. In the presence of an external magnetic field, the spins rotate about the axis of that field. $B_{0}$ is (approximately) spatially uniform, so all spins initially rotate at the same frequency (the Larmor frequency), $\omega=\gamma B_{0}$, where $\gamma$ is the gyromagnetic ratio $(\gamma=42.6 \mathrm{MHz} / \mathrm{Tesla}$ for hydrogen protons). However, when any of the gradients is played, the magnetic field becomes spatially varying, and so does the rotation frequency of the spins. Therefore, $G_{x}, G_{y}$, and $G_{z}$ are used to frequency-encode (or phase-encode) spatial position along the $x, y$ and $z$ directions, respectively.

The final major component of the MR scanner is the radio-frequency (RF) coil. This is used to transmit a RF "excitation" pulse to the body, and also to receive the frequency-encoded signal from the "excited" portion of the body. In practice, independent coils may be used for transmission and reception. The RF pulse is typically modulated to the Larmor frequency. While $B_{0}$ is aligned with the $z$-axis (by definition), $B_{1}$, which is a very weak magnetic field associated with the RF pulse, is aligned with the $x$-axis (also by definition). When the RF pulse is played, some of the spins which are in resonance with the RF pulse (i.e., rotating at the RF pulse's frequency) will now begin to rotate around the $x$-axis (thus the name magnetic resonance). This tilts the net magnetization towards the $x-y$ plane, and the net magnetization will now have a component in the $x-y$ plane $\left(M_{x y}\right)$.

The RF pulse is typically designed to have a somewhat rectangular profile in Fourier domain, centered at the modulation frequency (e.g., a modulated windowed sinc). This implies that the RF pulse in fact contains a certain range of frequencies, thus all spins rotating within that range become "excited", or tilted towards the $x$-axis. So, by playing gradient(s) of an appropriate amplitude, and designing the RF pulse accordingly, one can excite only a thin slice of the body, which correspond to the region containing all spins that are in resonance with the RF pulse's range of frequencies. Excitation profiles other than "slices" may also be obtained (e.g., a pencil beam, or cylindrical excitation (Hu et al., 1993)), by designing an appropriate gradient/pulse combination.

When the RF pulse is turned off, $M_{x y}$ begins to rotate (at the Larmor frequency) around the $z$-axis, as the net magnetization begins to realign with $B_{0}$. This rotating magnetization generates an oscillating signal, which can be detected by the receive coil. The frequency content of the received signal can be used to obtain spatial information about the excited portion of the body. In order to frequency-encode spatial information, gradients are also played during signal acquisition. These are called readout gradients. For imaging a slice perpendicular to the $z$-axis (an axial image), $G_{z}$ is played during excitation (for slice-selection), and $G_{x}$ and $G_{y}$ are played during acquisition. These can be switched, for acquiring sagittal or coronal images, or all three gradients may be used during both excitation and acquisition to image oblique planes. 
When the readout gradients are played, the acquired signal at a particular time instant corresponds to the sum of different sinusoidal signals generated by spins located at different regions of the body, each rotating at different frequencies corresponding to their spatial locations. If an axial slice is being acquired, for example, the demodulated signal value is equivalent to a sample of the Fourier transform $M\left(k_{x}, k_{y}\right)$ of the cross-sectional image $m(x, y)$. In this case, by changing the amplitudes of $G_{x}$ and $G_{y}$ during acquisition, one may acquire different samples of $M\left(k_{x}, k_{y}\right)$. In fact, by playing $G_{x}$ and/or $G_{y}$, one can move along the $k_{x}-k_{y}$ plane (which is known in MRI as k-space), collecting samples of $M\left(k_{x}, k_{y}\right)$. When enough samples of $M\left(k_{x}, k_{y}\right)$ have been collected, an inverse Fourier transform produces $m(x, y)$.

The required coverage of $\mathrm{k}$-space, and the number of samples, depend on the specified spatial resolution and field-of-view. For low spatial resolution imaging, only the central portion of $k_{x}-k_{y}$ needs to be sampled. For higher spatial resolution, the periphery of k-space must also be covered. The field of view is associated with the spacing between samples. For a larger field-of-view, k-space needs to be more densely sampled, requiring an increased number of samples. If k-space is not sufficiently sampled, and the resulting field-of-view is not large enough to cover the entire object, overlap in spatial domain is observed (aliasing).

Because signal amplitude is lost as the net magnetization realigns with $B_{0}$ (this is called relaxation), multiple acquisitions (excitation + readout) may be needed in order to cover the entire k-space. Different trajectories are more efficient in covering k-space than others. For example, spiral imaging, which uses oscillating gradients to achieve spiral k-space trajectories (Figure $1 b$ ), are generally faster than 2DFT imaging, i.e., require fewer acquisitions. In 2DFT imaging, each acquisition readout acquires a single line of k-space, sampling $k_{x}-k_{y}$ in a Cartesian fashion (Figure 1a). This is generally slower, but may be advantageous in some applications with respect to the nature of associated image artifacts. The fashion in which RF pulses and gradients are played is called pulse sequence. The time between acquisitions is called pulse repetition time, or TR.
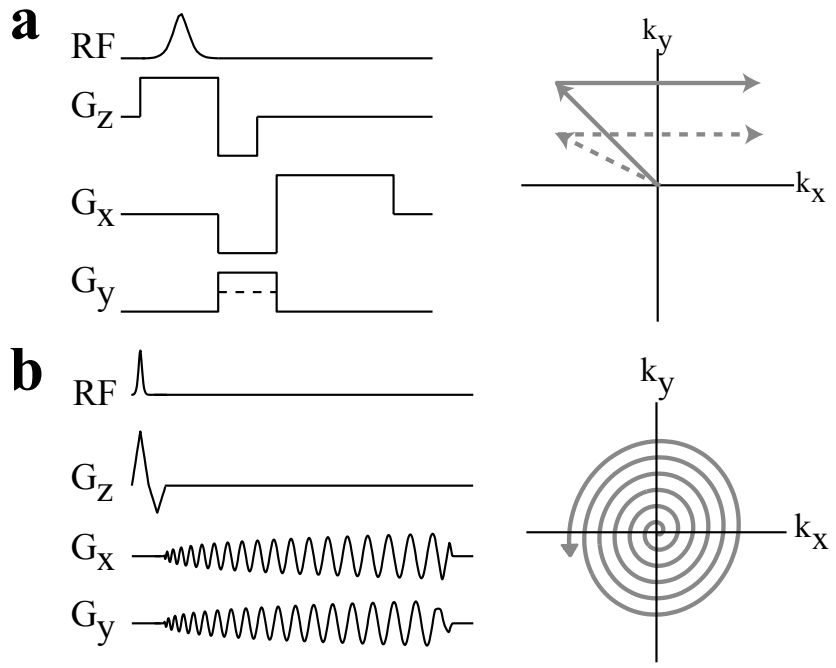

Fig. 1. Timing diagram (left) and corresponding k-space trajectories (right) for (a) 2DFT, and (b) spiral acquisitions. 


\subsection{Mathematical formalism}

As discussed on section 2.1, the acquired MR signal $s(t)$ at a particular time instant corresponds to a sample of the Fourier transform $M\left(k_{x}, k_{y}\right)$ of the cross-sectional image $m(x, y)$ :

$$
M\left(k_{x}, k_{y}\right)=\int_{x} \int_{y} m(x, y) e^{-j 2 \pi\left(k_{x} x+k_{y} y\right)} d x d y .
$$

The Fourier coordinates $k_{x}$ and $k_{y}$ vary with time, according to the zeroth moment of the readout gradients $G_{x}$ and $G_{y}$ :

$$
\begin{aligned}
& k_{x}(t)=\frac{\gamma}{2 \pi} \int_{0}^{t} G_{x}(\tau) d \tau \\
& k_{y}(t)=\frac{\gamma}{2 \pi} \int_{0}^{t} G_{y}(\tau) d \tau .
\end{aligned}
$$

These equations explain how the gradients can be used to "move" along k-space, as discussed on section 2.1. This formalism can be generalized for any combination of the gradients $G_{x}, G_{y}$ and $G_{z}$ as:

$$
\begin{aligned}
M\left(\vec{k}_{r}\right) & =\int_{\vec{r}} m(\vec{r}) \cdot e^{-j 2 \pi \vec{k}_{r} \cdot \vec{r}} d \vec{r} \\
\vec{k}_{r}(t) & =\frac{\gamma}{2 \pi} \int_{0}^{t} \vec{G}_{r}(\tau) d \tau,
\end{aligned}
$$

where $\vec{G}_{r}$ is the oblique gradient resulting from the combination of the $G_{x}, G_{y}$ and $G_{z}$ gradients, and $\vec{r}$ is its corresponding axis, along which the linear variation in magnetic field intensity is perceived.

Given a spatial position function $\vec{r}(t)$ and a magnetic field gradient $\vec{G}_{r}(t)$, the magnetization phase is:

$$
\phi(\vec{r}, t)=\gamma \int_{0}^{t} \vec{G}_{r}(\tau) \cdot \vec{r}(\tau) d \tau,
$$

For static spins, $\vec{r}(t)$ is constant $(\vec{r})$, and this becomes:

$$
\begin{aligned}
\phi & =\gamma \vec{r} \cdot \int_{0}^{t} \vec{G}_{r}(\tau) d \tau \\
& =2 \pi \vec{k}_{r} \cdot \vec{r},
\end{aligned}
$$

as in the exponential in equation 4.

\subsection{Principles of MR flow imaging}

The basic principles of quantitative flow measurement using magnetic resonance were first proposed by Singer (1959) and Hahn (1960) in the late 1950's. However, clinical applications of MR flow quantitation weren't reported until the early 1980's (Moran et al., 1985; Nayler et al., 1986; Singer \& Crooks, 1983; van Dijk, 1984). Current MR flow imaging methods are based on the fact that spins moving at a constant velocity accrue a phase proportional to the 
velocity times the first moment of the gradient waveform along the direction in which they are moving.

For spins moving along the $\vec{r}$-axis with a constant velocity $\vec{v}$, and initial position $\vec{r}_{0}$, we can write $\vec{r}(t)=\overrightarrow{r_{0}}+\vec{v} t$. Rewriting equation 6 , for $t=t_{0}$ :

$$
\begin{aligned}
\phi & =\gamma \int_{0}^{t_{0}} \vec{G}_{r}(t) \cdot\left(\vec{r}_{0}+\vec{v} t\right) d t \\
& =\gamma \vec{r}_{0} \cdot \int_{0}^{t_{0}} \vec{G}_{r}(t) d t+\gamma \vec{v} \cdot \int_{0}^{t_{0}} \vec{G}_{r}(t) t d t \\
& =\gamma \vec{r}_{0} \cdot \vec{M}_{0}+\gamma \vec{v} \cdot \vec{M}_{1},
\end{aligned}
$$

where $\vec{M}_{0}$ and $\vec{M}_{1}$ are the zeroth and first moments of the $\vec{r}$-gradient waveform at echo time, respectively. Thus, if a gradient with null zeroth moment is used (e.g., a bipolar gradient, aligned with $\vec{v}$ ), the phase accrued for a constant velocity spin is $\phi=\gamma \vec{v} \cdot \vec{M}_{1}$.

Therefore, if a bipolar gradient waveform is played between the excitation and the readout, the phase measured in a pixel of the acquired image is directly proportional to the velocity of the spins contained within its corresponding voxel. However, factors other than flow (such as inhomogeneities of the magnetic field) may cause additional phase shifts that would cause erroneous interpretation of the local velocity (Rebergen et al., 1993).

\subsubsection{Phase contrast}

The phase contrast method addresses the problem mentioned above by using two gradient-echo data acquisitions in which the first moment of the bipolar gradient waveform is varied between measurements (O'Donnell, 1985). The velocity in each voxel is measured as:

$$
v(x, y)=\frac{\phi_{a}(x, y)-\phi_{b}(x, y)}{\gamma\left(M_{1}^{a}-M_{1}^{b}\right)},
$$

where $\phi_{a}(x, y)$ and $\phi_{b}(x, y)$ are the phase images acquired in each acquisition, and $M_{1}^{a}$ and $M_{1}^{b}$ are the first moment of the bipolar gradients used in each acquisition.

\subsubsection{Fourier velocity encoding}

While phase contrast provides a single velocity measurement associated with each voxel, Fourier velocity encoding (FVE) (Moran, 1982) provides a velocity histogram for each spatial location, which is a measurement of the velocity distribution within each voxel.

FVE involves phase-encoding along a velocity dimension. Instead of only two acquisitions, as in phase contrast, multiple acquisitions are performed, and a bipolar gradient with a different amplitude (and first moment) is used in each acquisition. Equation 10 can be rewritten as:

$$
\phi(\vec{r}, \vec{v}, t)=2 \pi\left(\vec{k}_{r} \cdot \vec{r}+\vec{k}_{v} \cdot \vec{v}\right),
$$

where $\vec{k}_{v}$ is the velocity frequency variable associated with $\vec{v}$, and is proportional to the first moment of $\vec{G}_{r}(t)$ :

$$
\vec{k}_{v}=\frac{\gamma}{2 \pi} \vec{M}_{1}
$$


Each voxel of the two-dimensional image is associated with a distribution of velocities. This three-dimensional function, $m(x, y, v)$, is associated with a three-dimensional Fourier space, $M\left(k_{x}, k_{y}, k_{v}\right)$. Thus, an extra dimension is added to k-space, and multiple acquisitions are required to cover the entire $k_{x}-k_{y}-k_{v}$ space. In order to move along $k_{v}$, a bipolar gradient with the appropriate amplitude (and first moment) is played before the $k_{x}-k_{y}$ readout gradients, in each acquisition. Placing the bipolar gradient along the $z$-axis will encode through-plane velocities. Placing the bipolar gradient along $x$ or $y$ will encode in-plane velocities. Oblique flow can be encoded using a combination of bipolar gradients along the $x, y$ and $z$ axes.

Each acquisition along $k_{v}$ is called a velocity encode. The number of required velocity encodes depends on the desired velocity resolution and velocity field-of-view (the maximum range of velocities measured without aliasing). For example, to obtain a $25 \mathrm{~cm} / \mathrm{s}$ resolution over a $600 \mathrm{~cm} / \mathrm{s}$ field-of-view, 24 velocity encodes are needed. The velocity distributions along the cross-sectional image $m(x, y, v)$ is obtained by inverse Fourier transforming the acquired data $M\left(k_{x}, k_{y}, k_{v}\right)$. If cine imaging (Glover \& Pelc, 1988) is used, measurements are also time resolved, resulting in a four-dimensional dataset: $m(x, y, v, t)$.

The main drawback of FVE is scan time, as $k_{x}-k_{y}$ should be fully sampled for each value of $k_{v}$. As discussed in section 1.2.2, different approaches to accelerating FVE have been proposed. Those techniques are typically inefficient in spatially separating flowing blood from nearby static tissue. Furthermore, they are not capable of resolving multiple flows in a single acquisition. The spiral FVE method, proposed in section 4, addresses these limitations.

\section{Experimental setup}

Most experiments were performed on a Signa 3 T EXCITE HD system (GE Healthcare), with gradients capable of $40 \mathrm{mT} / \mathrm{m}$ amplitude and $150 \mathrm{~T} / \mathrm{m} / \mathrm{s}$ slew rate, and a receiver with sampling interval of $4 \mu \mathrm{s}$. Sequence designs were optimized for this scanner configuration. The body coil was used for RF transmission in all studies. An 8-channel phase array cardiac coil was used in the healthy volunteer studies, but data from only 1 or 2 elements were used in reconstruction. In phantom studies, a single channel 5-inch surface coil was used. In the patient experiments presented in section 4, a Signa 1.5 T LX system (GE Healthcare) with the same gradient and receiver configuration was used, and acquisition was performed using a 5 -inch surface coil.

The institutional review boards of the University of Southern California and Stanford University approved the imaging protocols. Subjects were screened for magnetic resonance imaging risk factors and provided informed consent in accordance with institutional policy.

\section{Slice-selective FVE with spiral readouts (spiral FVE)}

In order to address the limitations of existing flow imaging methods, we propose the use of slice-selective FVE MRI with spiral acquisitions. The proposed spiral FVE method is capable of acquiring fully localized, time-resolved velocity distributions in a short breath-hold. Scan-plane prescription is performed using classic protocols.

We present practical implementations for measuring blood flow through the aortic valve, and comparisons with Doppler ultrasound and high-resolution 2DFT phase contrast MRI. The proposed method is demonstrated in healthy volunteers and in a patient. 


\subsection{Pulse sequence}

The spiral FVE imaging pulse sequence (Figure 2) consists of a slice-selective excitation, a velocity-encoding bipolar gradient, a spiral readout, and refocusing and spoiling gradients. The dataset corresponding to each temporal frame is a stack-of-spirals in $k_{x}-k_{y}-k_{v}$ space (Figure 3). The bipolar gradient effectively phase-encodes in $k_{v}$, while each spiral readout acquires one "disc" in $k_{x}-k_{y}$.

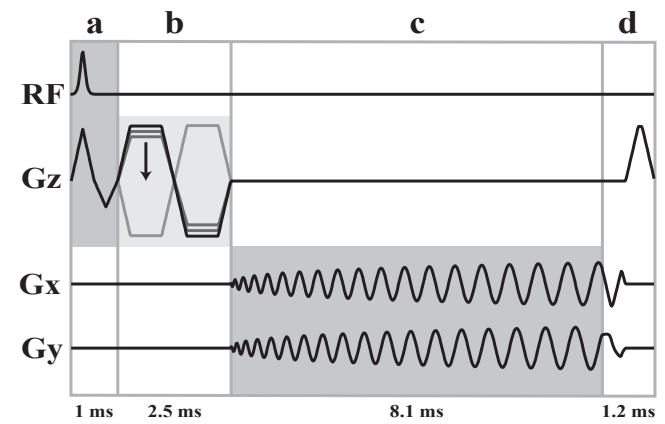

Fig. 2. Spiral FVE pulse sequence. It consists of (a) slice selective excitation, (b) velocity encoding bipolar gradient, (c) spiral readout, and (d) refocusing and spoiling gradients. This timing corresponds to the studies shown in Figures 5 and 6.

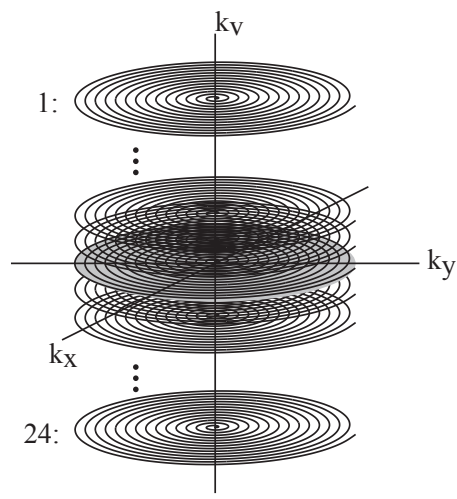

Fig. 3. Spiral FVE k-space sampling scheme. The dataset corresponding to each temporal frame is a stack-of-spirals in $k_{x}-k_{y}-k_{v}$ space. Each spiral acquisition corresponds to a different $k_{v}$ encode level.

\subsection{Signal model}

2DFT phase contrast provides two two-dimensional functions, $m(x, y)$ and $v_{o}(x, y)$, the magnitude and velocity maps, respectively. If these maps are measured with sufficiently high spatial resolution, and flow is laminar, one can assume that each voxel contains only one velocity, and therefore the spatial-velocity distribution associated with the object is approximately:

$$
s(x, y, v)=m(x, y) \times \delta\left(v-v_{o}(x, y)\right),
$$


where $\delta(v)$ is the Dirac delta function.

As spiral FVE acquisitions follow a stack-of-spirals pattern in $k_{x}-k_{y}-k_{v}$ space (Figure 3), $\mathrm{k}$-space data is truncated to a cylinder, i.e., a circle along $k_{x}-k_{y}$ (with diameter $1 / \Delta r$ ), and a rectangle along $k_{v}$ (with width $1 / \Delta v$ ), where $\Delta r$ and $\Delta v$ are the prescribed spatial and velocity resolutions, respectively. The associated object domain spatial-velocity blurring can be modeled as a convolution of the true object distribution, $s(x, y, v)$, with jinc $\left(\sqrt{x^{2}+y^{2}} / \Delta r\right)$ and $\operatorname{sinc}(v / \Delta v)$, resulting in:

$$
\begin{aligned}
\hat{s}(x, y, v) & =\left[m(x, y) \times \delta\left(v-v_{o}(x, y)\right)\right] * \operatorname{sinc}\left(\frac{v}{\Delta v}\right) * \operatorname{jinc}\left(\frac{\sqrt{x^{2}+y^{2}}}{\Delta r}\right) \\
& =\left[m(x, y) \times \operatorname{sinc}\left(\frac{v-v_{o}(x, y)}{\Delta v}\right)\right] * \operatorname{jinc}\left(\frac{\sqrt{x^{2}+y^{2}}}{\Delta r}\right)
\end{aligned}
$$

where $\hat{s}(x, y, v)$ is the measured object distribution, and $*$ denotes convolution.

\subsection{Data acquisition}

The excitation achieved a $5 \mathrm{~mm}$ slice thickness and $30^{\circ}$ flip angle, with a $0.5 \mathrm{~ms}$ RF pulse and a $1 \mathrm{~ms}$ gradient. Through-plane velocity encoding was implemented using a large bipolar pulse along the $z$ direction that was scaled to achieve different $k_{v}$ encodes. The velocity resolution is determined by the first moment of the largest bipolar gradient, and the velocity field-of-view is determined by the increment in gradient first moment for different velocity encodes. A bipolar duration of $2.5 \mathrm{~ms}$ achieves a velocity resolution of $25 \mathrm{~cm} / \mathrm{s}$. Gradient duration increases if velocity resolution is improved.

An $8.1 \mathrm{~ms}$ optimized uniform-density single-shot spiral acquisition (Hargreaves, 2001) acquires $k_{x}-k_{y}$ at each velocity encode, and zeroth and first moments are refocused in $0.5 \mathrm{~ms}$. The readout and refocusing gradients were designed using public domain software ${ }^{1}$. A 0.65 ms spoiling gradient (Zur et al., 1991) achieves a $6 \pi$ phase-wrap over the slice thickness. The spoiling gradient was not overlapped with the refocusing gradients, but this could be done to further shorten the TR. The minimum TR (approximately $13 \mathrm{~ms}$ ) was used in all studies. Other scan dependent pulse sequence parameters are listed in Table 1.

Prospective ECG gating was used to synchronize acquisitions with the cardiac cycle. Two $k_{v}$ levels were repeatedly acquired during each heartbeat in order to resolve 25 to 35 cardiac phases and produce a cine dataset (Figure 5, discussed later). The true temporal resolution was $26 \mathrm{~ms}$ (2 TRs). Sliding window reconstruction (Riederer et al., 1988) was used to produce a new image every $13 \mathrm{~ms}$.

\subsection{Data reconstruction}

Reconstruction was performed in Matlab (The MathWorks, Inc., Natick, MA, USA). Each spiral interleaf is first gridded (Jackson et al., 1991) and inverse Fourier transformed to form an image, $m(x, y)$, for each temporal frame. This step converts the acquired data $S_{k_{x}, k_{y}}\left(k_{v}, t\right)$ to $S_{x, y}\left(k_{v}, t\right)$. The operator manually defines a region of interest (ROI) in the $x-y$ plane using the image corresponding to $k_{v}=0$ and $t=0$. Pixel intensities within the ROI are averaged at each temporal frame, resulting in a $2 \mathrm{D}$ dataset: $S_{\mathrm{ROI}}\left(k_{v}, t\right)=\sum_{x, y}^{\mathrm{ROI}} S_{x, y}\left(k_{v}, t\right)$. View sharing is

\footnotetext{
${ }^{1}$ http:/ / www - mrsrl.stanford.edu/ brian/vdspiral/
} 


\begin{tabular}{lcc}
\hline & healthy volunteer & patient \\
\hline field-of-view & $25 \mathrm{~cm}$ & $20 \mathrm{~cm}$ \\
spatial resolution & $7 \mathrm{~mm}$ & $6.5 \mathrm{~mm}$ \\
& & \\
$k_{v}$ encodes & 24 & 64 \\
velocity field-of-view & 600 or $800 \mathrm{~cm} / \mathrm{s}$ & $1200 \mathrm{~cm} / \mathrm{s}$ \\
velocity resolution & 25 or $33 \mathrm{~cm} / \mathrm{s}$ & $19 \mathrm{cms} / \mathrm{s}$ \\
& & \\
$k_{v}$ encodes/heartbeat & 2 & 4 \\
TR & 12.8 or $12.5 \mathrm{~ms}$ & $12.8 \mathrm{~ms}$ \\
temporal resolution & 25.6 or $25 \mathrm{~ms}$ & $51.2 \mathrm{~ms}$ \\
& & \\
scan time & 12 heartbeats & 16 heartbeats
\end{tabular}

Table 1. Scan parameters used in the different spiral FVE studies.

then applied to $S_{\mathrm{ROI}}\left(k_{v}, t\right)$ to increase the number of temporal frames (Riederer et al., 1988). Saturation effects (Gao et al., 1988) are compensated by normalizing the $\ell_{2}$-norm of $S_{\text {ROI }}\left(k_{v}\right)$ independently for each temporal frame, which effectively normalizes each cardiac phase. $S_{\mathrm{ROI}}\left(k_{v}, t\right)$ is then zero-padded along the $k_{v}$ axis, and an inverse Fourier transform produces $s_{\mathrm{ROI}}(v, t)$. The time-velocity histogram for the ROI is $\left|s_{\mathrm{ROI}}(v, t)\right|$, and for display purposes, smoother histograms are obtained by cubic spline interpolation along $t$ (Bartels et al., 1987). The reconstruction process can be repeated for each voxel, or for multiple regions of interest, using the same data (see Figure 8, discussed later).

\subsection{Accuracy of spiral FVE measurements}

An in vitro comparison of velocity distributions measured with spiral FVE with those derived from high-resolution 2DFT phase contrast - the current MR gold standard - was performed. The signal model presented on section 4.2 was used to generate simulated FVE data based on high-resolution 2DFT phase contrast data.

The validation experiments were performed using a pulsatile carotid flow phantom (Phantoms by Design, Inc., Bothell, WA). A slice perpendicular to the phantom's carotid bifurcation was prescribed, and through-plane velocities were measured. A cine gradient-echo 2DFT phase contrast sequence with high spatial resolution and high SNR ( $0.33 \mathrm{~mm}$ resolution, 10 averages, $80 \mathrm{~cm} / \mathrm{s}$ Venc) was used as a reference. Cine spiral FVE data with $\Delta r=3 \mathrm{~mm}$ and $\Delta v=10 \mathrm{~cm} / \mathrm{s}$ was obtained from the same scan plane. Both acquisitions were prospectively gated, and used the same TR $(11.6 \mathrm{~ms})$, flip angle $\left(30^{\circ}\right)$, slice profile $(3 \mathrm{~mm})$, temporal resolution $(23.2 \mathrm{~ms})$, and pre-scan settings. The total scan time was 40 minutes for phase contrast, and 12 seconds for FVE.

A simulated spiral FVE dataset was computed from the PC magnitude and velocity maps, using the convolution model described in Eq. 16. The PC-derived and FVE-measured data were registered by taking one magnitude image $m(x, y)$ from each dataset, and then using the phase difference between their Fourier transforms $M\left(k_{x}, k_{y}\right)$ to estimate the spatial shift between the images. Amplitude scaling was performed by normalizing the $\ell_{2}$-norm of each FVE dataset. The difference between PC-derived and FVE-measured time-velocity distributions was calculated for select voxels, and the associated signal-to-error ratios were computed. This was used as a quantitative assessment of spiral FVE's accuracy. 
Figure 4 shows measured and PC-derived time-velocity FVE distributions from nine representative voxels, selected around the circumference of the vessel wall of the pulsatile carotid flow phantom's bifurcation. The signal-to-error ratio between measured and PC-derived time-velocity distributions was measured to be within 9.3-11.7 dB. Imperfect registration between the datasets, combined with spatial blurring due to off-resonance in the measured spiral FVE data, may have contributed to this moderate signal-to-error ratio. Nevertheless, the two datasets show good visual agreement, and no significant spatial variation was observed in terms of accuracy. These results show that velocity distributions measured with spiral FVE agree well with those obtained with 2DFT phase contrast, the current MRI gold standard. This approach for deriving FVE data from high-resolution velocity maps (Eq. 16) can be used for many simulation purposes (Carvalho et al., 2010).
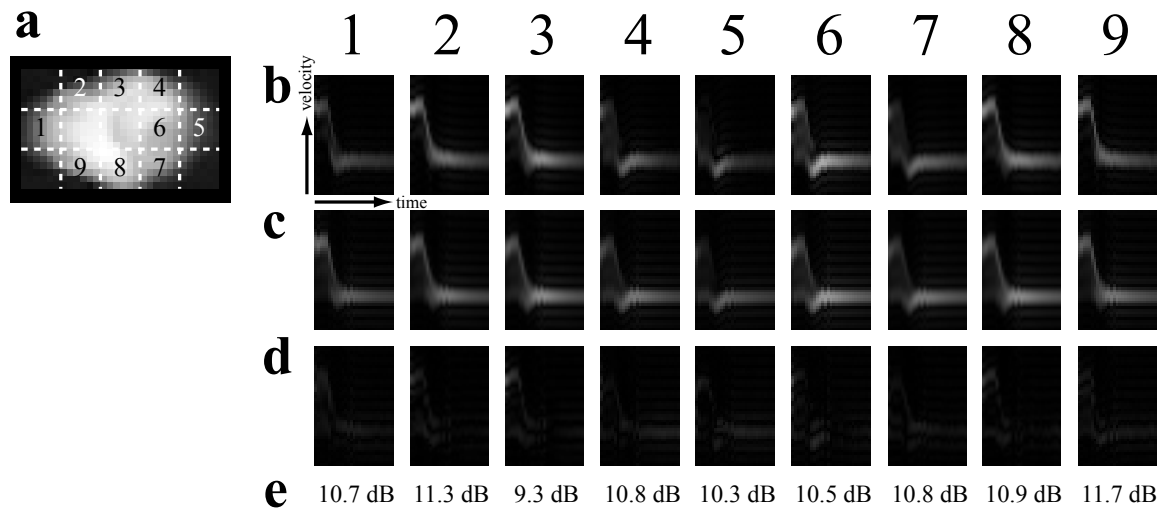

Fig. 4. In vitro evaluation of the accuracy of spiral FVE velocity histograms. Results are shown for nine representative voxels, selected around the circumference of the vessel wall of the pulsatile carotid flow phantom's bifurcation (a). For each voxel, it is shown: (b) time-velocity distribution derived from high-resolution 2DFT phase contrast; (c) time-velocity distribution measured with spiral FVE; (d) absolute difference between spiral FVE and 2DFT PC-derived histograms; (e) signal-to-error ratio.

\subsection{Aortic valve flow assessment using spiral FVE}

The proposed method was evaluated in vivo, aiming at quantifying flow through the aortic valve. Scan-plane prescription was performed using a real-time imaging sequence.

For a severely stenosed heart valve, peak velocities can reach up to $600 \mathrm{~cm} / \mathrm{s}$ (Galea et al., 2002). As regurgitant jets don't overlap in time with forward flow, we used a $600 \mathrm{~cm} / \mathrm{s}$ velocity field-of-view $( \pm 300 \mathrm{~cm} / \mathrm{s})$. This value could be increased by extending the scan time, or by sacrificing temporal, velocity and/or spatial resolutions (see Figure 9, discussed later). Scan parameters were summarized in Table 1.

\subsubsection{Order of velocity encode acquisitions}

When the $k_{v}$ levels are acquired in a sequential fashion, ghosting artifacts due to data inconsistency appear shifted by one half of the velocity field-of-view (Figure 5). Using this sampling scheme and an appropriate velocity field-of-view, the artifacts will not overlap with the flow profile and may be easily identified and masked out. 


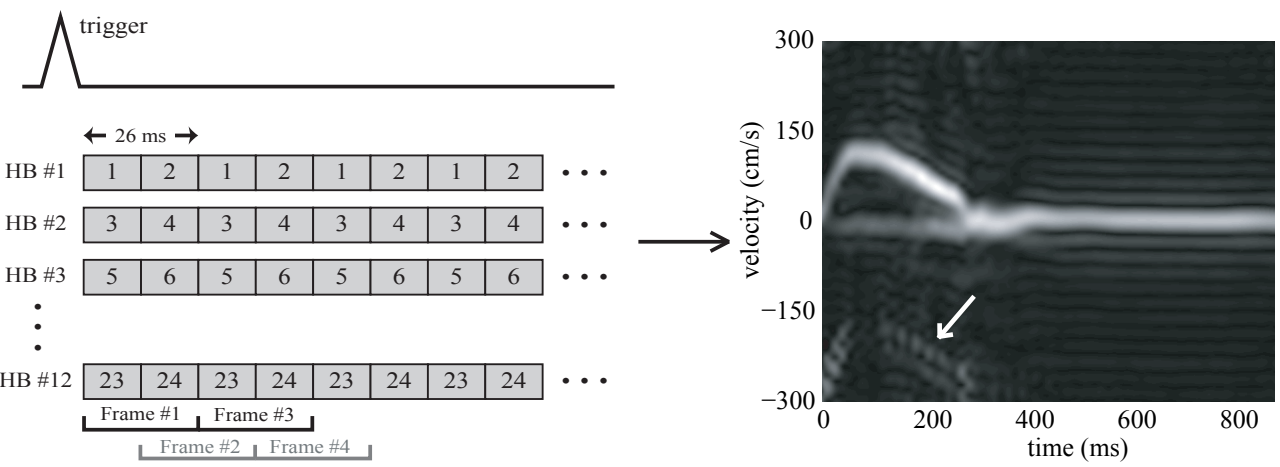

Fig. 5. Artifacts with sequential view-ordering scheme. Each box represents the acquisition of one $k_{v}$ level, during one imaging TR. A sliding window is used to produce a new image every TR. Ghosting artifacts appear shifted by $1 / 2$ of the velocity field-of-view when the $k_{v}$ levels are acquired in this sequential fashion (see white arrow).

Artifacts and loss of temporal resolution due to view sharing can be avoided or corrected using different approaches. Acquiring multiple $k_{v}$ levels per heartbeat reduces scan time, but causes blurring along the time axis and ghosting along the velocity axis. Blurring is caused by the reduction in temporal resolution, and ghosting artifacts arise when the velocity distribution changes between the acquisition of consecutive velocity encodes. Both ghosting and blurring can be overcome by acquiring only one view per heartbeat, but this would require increase in scan time or reduction in velocity resolution. As an alternative, these artifacts may be corrected using techniques that exploit efficient use of $k-t$ space, such as the approach proposed in section 5 .

\subsubsection{Spiral FVE vs. doppler ultrasound}

A representative in vivo result is compared with Doppler ultrasound in Figure 6. The MRI measured time-velocity histogram show good agreement with the ultrasound measurement, as the peak velocity and the shape of the flow waveform were comparable to those observed in the ultrasound study.
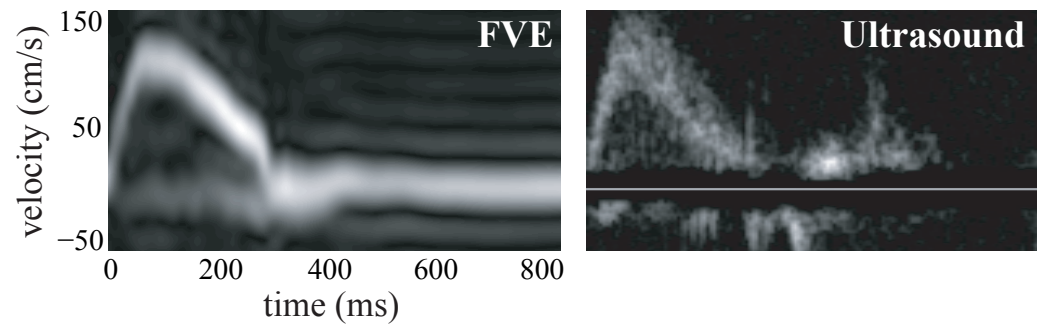

Fig. 6. Comparison of the spiral FVE method with Doppler ultrasound, in a healthy volunteer aortic valve study. 


\subsubsection{Patient evaluation}

Figure 7 shows the time-velocity distribution measured through the aortic valve of a patient with aortic stenosis. This result demonstrate that spiral FVE can accurately detect complex flow, as a high-speed jet with a wide distribution of velocities is clearly visible.

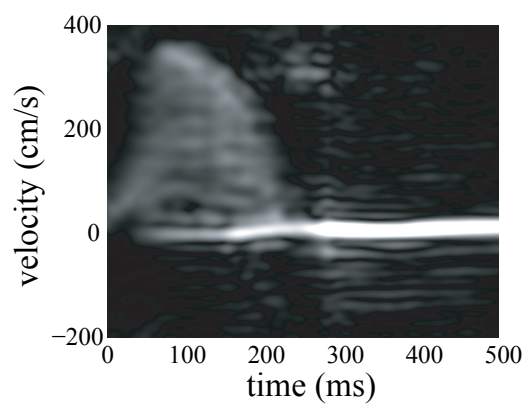

Fig. 7. Evaluation of spiral FVE in a patient with aortic stenosis. Note the high-speed jet with a wide distribution of velocities.

\subsubsection{Measurement of multiple flows}

Figure 8 illustrates spiral FVE's ability of resolving different flows from a single dataset. A different flow distribution was calculated for each voxel, and the distributions from single voxels from different ROIs are displayed. Red and blue dots indicate voxels where ascending and descending blood flows were detected, respectively, and the color intensity of each dot indicates the highest velocity detected in that voxel in a particular temporal frame.

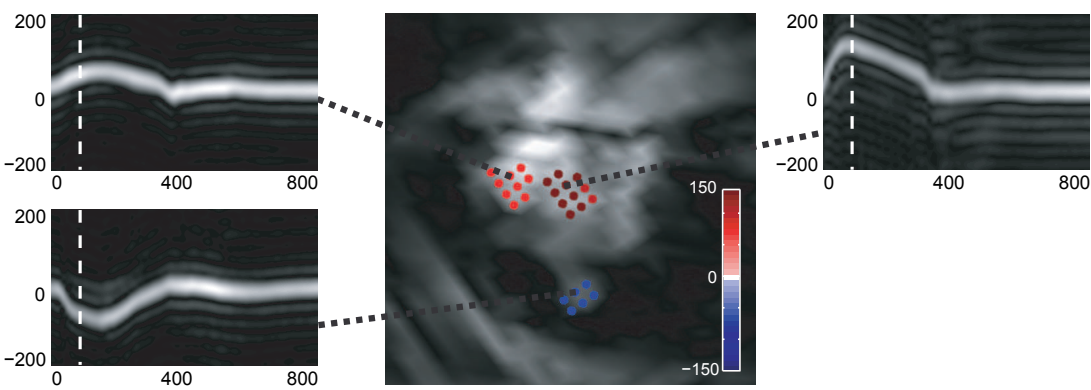

Fig. 8. Multiple flow distributions obtained from a single spiral FVE dataset. For each voxel in the image, a flow distribution was calculated, and the red and blue dots indicate voxels where ascending and descending blood flows were detected, respectively. The color intensity of each dot indicates the highest velocity detected in that voxel in a particular temporal frame (indicated by the white dashed lines).

\subsection{Resolution trade-offs}

In spiral FVE, there is an important trade-off between velocity resolution, temporal resolution, and scan time (Figure 9). This trade-off also involves other scan parameters, such as velocity field-of-view, number of spiral interleaves, spiral readout duration, spatial resolution, and spatial field-of-view. Velocity resolution can be improved in many ways, such as increasing 
the breath-hold duration to acquire more $k_{v}$ levels, or by reducing the velocity field-of-view. Temporal resolution can be made as high as one TR duration (13 ms) by segmenting the $k_{v}$ encodes across additional heartbeats (longer breath-holds), or by compromising velocity resolution or field-of-view. Spatial resolution can be improved by reducing the spatial field-of-view, or by increasing the number of spiral interleaves, which would require compromising other scan parameters such as scan time, temporal resolution and/or velocity resolution.

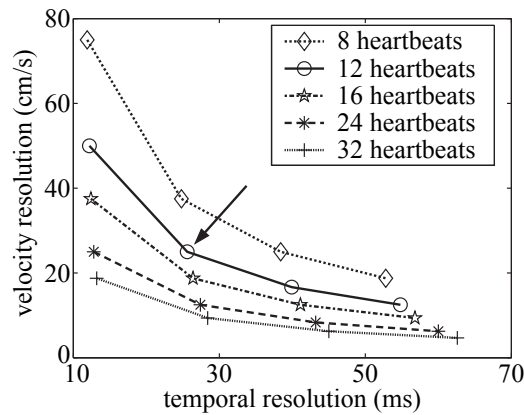

Fig. 9. Spiral FVE trade-offs between temporal resolution, velocity resolution, and breath-hold duration. Velocity resolution corresponds to a $600 \mathrm{~cm} / \mathrm{s}$ field-of-view, temporal resolution corresponds to a $8.1 \mathrm{~ms}$ spiral readout, and scan time corresponds to a single-shot spiral acquisition. The arrow indicates the configuration used in the study in Figure 6 (2.TR temporal resolution, 24 velocity encodes).

\subsection{Issues with spiral FVE}

As the spiral readouts are considerably long, a potential issue in spiral FVE imaging is blurring in image domain, due to off-resonance. Because SNR was not a limiting issue for the applications we have presented, spiral FVE may perform better at lower field strengths where there is reduced off-resonance. At $3 \mathrm{~T}$, localized shimming and off-resonance correction techniques can be used to reduce blurring. Furthermore, readout duration can be reduced by decreasing the spatial resolution or field-of-view, or by using variable-density spirals (Tsai \& Nishimura, 2000). Another alternative is to use multiple short spiral interleaves, which would require longer scan times, but parallel imaging techniques (Pruessmann et al., 2001; Samsonov et al., 2006) can potentially accelerate acquisition if multi-channel receiver coils are used. This approach also has the benefit of allowing increase in the frame rate, as the number of imaged cardiac phases is limited by the minimum TR. Another possible solution to the off-resonance problem is the use of echo-planar imaging trajectories, which produce different off-resonance effects (geometric warping) (Feinberg \& Oshio, 1992), but are also more sensitive to artifacts from in-plane flow or motion.

A noticeable artifact in spiral FVE is Gibbs ringing along the velocity dimension. These artifacts can be less noticeable if velocity resolution is increased, which would also improve the ability to visualize features in the flow waveform and the precision to resolve the peak velocity, but would require longer breath-holds. Alternatively, the velocity resolution can be improved by using variable-density sampling along $k_{v}$ (Carvalho et al., 2007; DiCarlo et al., 2005), or partial Fourier techniques (Noll, Nishimura \& Macovski, 1991). Another approach to reducing ringing artifacts is to window the $k_{v}$ samples before applying the inverse 
Fourier transform (Bernstein et al., 2001). However, windows with lower sidelobes generally have wider mainlobes, which would cause blurring along the velocity axis and consequent reduction in velocity resolution.

One drawback of the proposed method is the requirement of cardiac gating and breath-holding. Cardiac gating does not work well in patients with arrhythmias, and breath-holding may cause hemodynamic changes and is not possible for some patients (Macgowan et al., 2005). However, arrhythmia rejection (Chia et al., 2000) and respiratory gating schemes may overcome these problems, at the cost of increased scan time.

\section{Accelerated spiral FVE}

As introduced in section 4, spiral FVE presents limitations such as insufficient velocity field-of-view (FOV), low spatial resolution, and moderate temporal resolution. In particular, the use of view sharing (Riederer et al., 1988) causes blurring along the temporal dimension $(t)$ and ghosting along the velocity dimension $(v)$ (Figure 5), and the use of long spiral readouts makes the technique sensitive to off-resonance, resulting in blurring along the in-plane spatial axes $(x$ and $y$ ). The approach proposed in this section aims to address these limitations.

Spiral FVE datasets are four-dimensional, which makes this method particularly suitable for accelerated acquisition (Hansen et al., 2004). In this section, we achieve 18-fold acceleration using a combination of three techniques: variable-density spiral sampling along $k_{x}-k_{y}$ (Tsai \& Nishimura, 2000); partial Fourier (Noll, Nishimura \& Macovski, 1991) along $k_{v}$; and temporal acceleration through a novel implementation of the UNFOLD method (Madore et al., 1999; Tsao, 2002). The improved acquisition is performed without increase in scan time compared with the original implementation, and is demonstrated in vivo in a healthy volunteer.

\subsection{Accelerated data acquisition}

\subsubsection{Acceleration via variable-density sampling}

Variable-density spirals have been shown to increase spatiotemporal resolution and improve accuracy in flow quantitation (Liu et al., 2008). The spatial aliasing resulting from variable-density spiral sampling is incoherent, and, in the regions-of-interest (e.g., cardiac chambers, valves, great vessels), it typically originates from static or slow moving material located at the periphery of the spatial FOV (e.g., chest wall). FVE resolves the distribution of velocities within the voxel, thus moderate low-velocity aliasing artifacts generally do not affect one's ability to calculate diagnostically important parameters - such as peak velocity and acceleration - from the time-velocity distribution. Spiral FVE's single-shot uniform-density spiral readout was replaced with a multi-shot variable-density spiral acquisition (Tsai \& Nishimura, 2000). The use of multi-shot acquisitions provides the possibility of multi-dimensional temporal acceleration, and allows reduction of readout duration and TR, which reduce off-resonance artifacts and temporal aliasing, respectively. The use of a shorter TR also allows improving the temporal resolution. Gradient waveforms were designed using public domain software ${ }^{2}$, based on the hardware limits of our scanner. The spatial FOV was varied linearly from $25 \mathrm{~cm}$ at the center of k-space to $6.25 \mathrm{~cm}$ at the periphery.

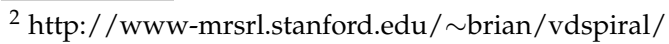




\subsubsection{Acceleration via UNFOLD}

Scan time in FVE imaging can be significantly reduced using multi-dimensional temporal acceleration (Gamper et al., 2008; Hansen et al., 2004). An implementation of the UNFOLD method (Madore et al., 1999; Tsao, 2002) was specially designed for spiral FVE. A view-ordering scheme that reduces overlap in $v-f$ space ( $f$ denotes temporal frequency) was designed. It consists in alternating spiral interleaves and $k_{v}$ encodings for each cardiac phase, according to Figure 10a. The associated point spread function is such that aliasing replicas, caused by temporal undersampling, are separated from the main lobe both in velocity (by half of the velocity FOV) and in temporal frequency (by 1/2TR) (Figure 10b) (Hansen et al., 2004; Tsao, 2002). Aliasing components caused by the sidelobes at \pm 20 and $\pm 40 \mathrm{~Hz}$ are expected to correspond to static or slow moving spins, and hence will have a small footprint in $v-f$ space. This is because these sidelobes spread around the periphery of the spatial FOV, but are null at the center, where high-velocity pulsatile flow is located. The aliasing signal is filtered using the two-dimensional filter shown in Figure 11. This filter has a bandwidth of $107 \mathrm{~Hz}$ for velocities below $\pm 150 \mathrm{~cm} / \mathrm{s}$. For higher velocities, the bandwidth varies from 69 to $30 \mathrm{~Hz}$. This results in effective temporal resolutions of $9.3 \mathrm{~ms}$ and $14.5-33.3 \mathrm{~ms}$, respectively. The temporal resolution is lower for higher velocities, but this may prove unnoticeable, as the velocity distribution of high-velocity flow jets within large voxels is typically temporally smooth. For comparison, the temporal resolution with the conventional approach - view sharing (Riederer et al., 1988) - would be $50 \mathrm{~ms}$ for all $v$. The remaining narrow-bandwidth aliasing components at \pm 20 and $\pm 40 \mathrm{~Hz}$ are filtered using a tight zero-phase one-dimensional notch filter along $t$.
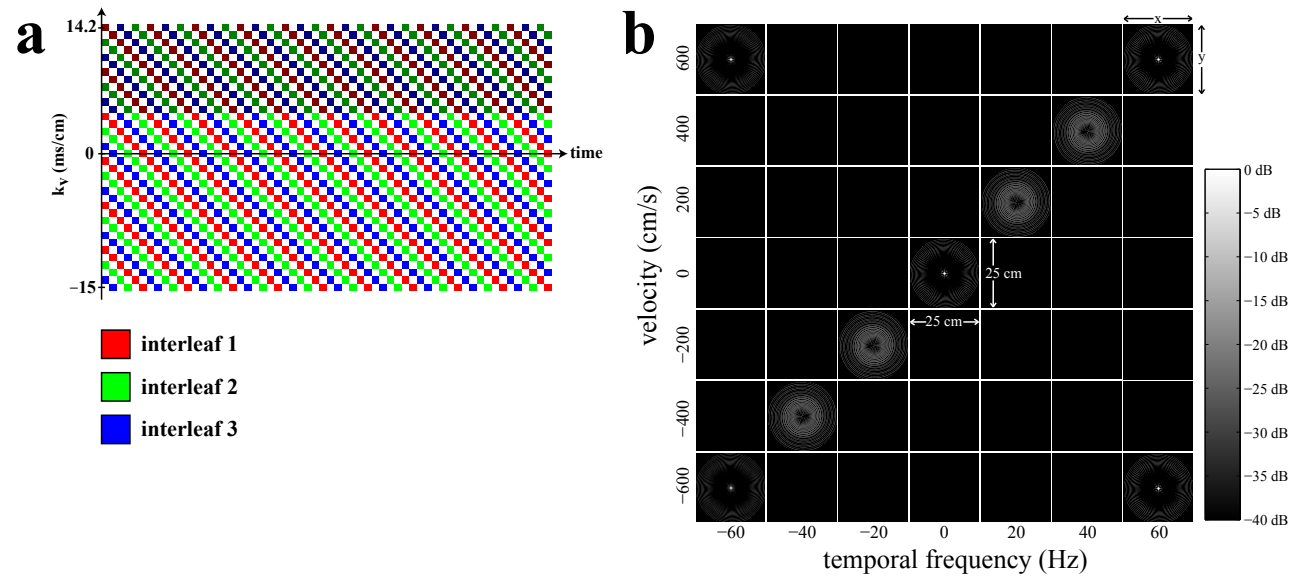

Fig. 10. Proposed view-ordering scheme for accelerated spiral FVE (a) and its corresponding point spread function (b). In (a), each color represents a different spiral interleaf, and darker tones indicate "views" that are discarded in the partial Fourier experiments. Views aligned in $k_{v}$ are acquired sequentially throughout the cardiac cycle. Views aligned in time (same cardiac phase) are acquired in different heartbeats. In (b), each square shows the point spread function in $x-y$ for a particular $v-f$ coordinate. Aliasing replicas are separated from the main lobe by half of the velocity FOV, and half of the temporal frequency bandwidth, which reduces overlaps and facilitates filtering. 


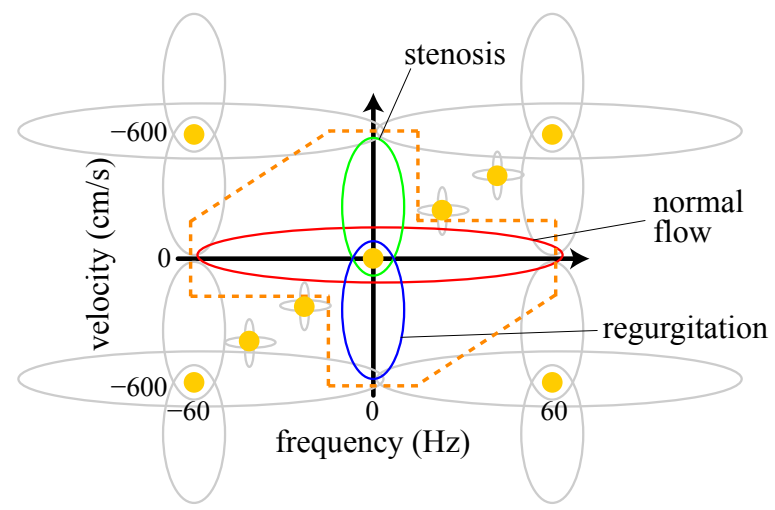

Fig. 11. Aliasing in $v-f$ space as a result of temporal undersampling. The red, green and blue ellipses illustrate the expected footprints for aortic valve flow for normal, stenotic, and regurgitant flows, respectively. Yellow dots represent peaks in the point spread function for the proposed undersampling scheme (see Figure 10). Grey ellipses represent potential aliasing components. Replicas at $\pm 60 \mathrm{~Hz}$ are exact copies of the true signal. The potential aliasing at \pm 20 and $\pm 40 \mathrm{~Hz}$ have a small footprint, because they are composed of signal from the periphery of the spatial FOV, i.e. static tissue or slow moving flow. A 2D filter (dashed lines) is capable of avoiding aliasing while preserving all signal content.

\subsubsection{Acceleration via partial Fourier}

Partial Fourier along the velocity dimension has been successfully used in FVE for scan time reduction, without significant loss of velocity resolution. This approach has been previously demonstrated in studies with healthy volunteers (Carvalho \& Nayak, 2007; Macgowan et al., 2005) and patients (Carvalho \& Nayak, 2007; Santos et al., 2007), and in phantom experiments (DiCarlo et al., 2005). Data was acquired with full coverage of $k_{v}$ space, and $33 \%$ of the data was retrospectively discarded before reconstruction (dark-colored squares in Figure 10a). The missing data was synthesized using homodyne reconstruction (Noll, Nishimura \& Macovski, 1991).

\subsection{Data reconstruction}

Reconstruction was performed in Matlab (The MathWorks, Inc., Natick, MA, USA). The acquired data, $S\left(k_{x}, k_{y}, k_{v}, t\right)$, is first re-sampled onto a Cartesian grid (Jackson et al., 1991) using a Kaiser-Bessel kernel designed for the largest FOV $(25 \mathrm{~cm})$. Each spiral interleaf is gridded separately, and inverse Fourier transformed to form a spatial image for its corresponding $k_{v}-t$ coordinate, resulting in $S\left(x, y, k_{v}, t\right)$. The data corresponding to the two central $k_{v}$ values $\left(k_{v}=0\right.$ and $\left.k_{v}=\frac{1}{\operatorname{Fov}_{v}}\right)$ are separately filtered using a 6-tap moving average temporal filter that effectively implements view sharing (Riederer et al., 1988). A color-flow video (Riederer et al., 1991) is obtained from the filtered data. The operator draws one or multiple ROIs over the video. Pixel values within each ROI are averaged, resulting in multiple 2D datasets: $S_{\mathrm{ROI}_{i}}\left(k_{v}, t\right)=\sum_{x, y}^{\mathrm{ROI}} S\left(x, y, k_{v}, t\right)$. Each of these 2D datasets is filtered using the 2D filter and the notch filter described in section 5.1.2. Saturation effects (Gao et al., 1988) are compensated by normalizing the data in each cardiac phase. The data is then zero-padded along the $k_{v}$ axis, and homodyne reconstruction (Noll, Nishimura \& Macovski, $1991)$ is used to produce each $s_{\mathrm{ROI}_{i}}(v, t)$ distribution. The time-velocity histogram for each 
$\mathrm{ROI}$ is $\left|S_{\mathrm{ROI}_{i}}(v, t)\right|$, and smoother histograms are generated by one-dimensional cubic spline interpolation (Bartels et al., 1987) along $t$.

\subsection{Acceleration experiments}

The use of variable-density sampling in spiral FVE for improving spatial resolution and reducing off-resonance artifacts was evaluated in the following experiment. Three acquisitions were performed, measuring flow at the aortic valve plane of a healthy volunteer. A different spiral design was used in each acquisition (Table 2). A reduced velocity FOV $(200 \mathrm{~cm} / \mathrm{s})$ was used, in order to limit each acquisition to a single feasible breath-hold. The FOV was adjusted to either the -50 to $150 \mathrm{~cm} / \mathrm{s}$ range or the -150 to $50 \mathrm{~cm} / \mathrm{s}$ range, depending on the flow of interest. Six $k_{v}$ encoding steps were acquired, resulting in a velocity resolution of $33 \mathrm{~cm} / \mathrm{s}$. No temporal undersampling was performed, and the acquisition was segmented across multiple heartbeats. The temporal resolution was one TR. The results were qualitatively compared in spatial and time-velocity domains.

\begin{tabular}{|c|c|c|c|}
\hline & $\begin{array}{c}\text { original } \\
\text { design }\end{array}$ & $\begin{array}{c}\text { proposec } \\
\text { design }\end{array}$ & $\begin{array}{l}\text { round truth } \\
\text { reference }\end{array}$ \\
\hline interleaves & 1 & 3 & 6 \\
\hline sampling & UD & VD & UD \\
\hline readout & $8 \mathrm{~ms}$ & $4 \mathrm{~ms}$ & $4 \mathrm{~ms}$ \\
\hline field-of-view & $25 \mathrm{~cm}$ & $6-25 \mathrm{~cm}$ & $25 \mathrm{~cm}$ \\
\hline resolution & $7.2 \mathrm{~mm}$ & $3.6 \mathrm{~mm}$ & $3.6 \mathrm{~mm}$ \\
\hline heartbeats $^{\dagger}$ & 6 & 18 & 36 \\
\hline $\mathrm{TR}^{\ddagger}$ & $12.4 \mathrm{~ms}$ & $8.4 \mathrm{~ms}$ & $8.4 \mathrm{~ms}$ \\
\hline
\end{tabular}

$\mathrm{UD}=$ uniform-density; $\mathrm{VD}=$ variable-density; ${ }^{\dagger} \mathrm{scan}$ time with $6 k_{v}$ encodes; ${ }^{\ddagger} \mathrm{TR}$ for $33 \mathrm{~cm} / \mathrm{s}$ velocity resolution.

Table 2. Design parameters used to evaluate the use of variable-density sampling in spiral FVE.

The proposed temporal acceleration scheme was then evaluated in a second experiment. Aortic valve flow was measured using the proposed variable-density spiral design (Table 2), and the proposed view-ordering scheme (Figure 10a). The velocity resolution and FOV were set to 33 and $1200 \mathrm{~cm} / \mathrm{s}$, respectively. The data was acquired in an 18-heartbeat breath-hold, and was reconstructed using view sharing, the proposed 2D filter, and the proposed notch filter after 2D-filtering. The reduced velocity FOV results from the previous experiment were used as ground truth reference for a qualitative comparison.

Partial Fourier acceleration was evaluated by discarding, before reconstruction, 12 of the $36 k_{v}$ encodes from the temporally-undersampled data from the previous experiment, as indicated in Figure 10a. The reconstructed time-velocity distribution was qualitatively compared with the fully sampled reference.

\subsection{Acceleration results}

Figure 12 contains results from the experiment using different spiral designs from Table 2 . The data in Figure 12a was obtained using the $8 \mathrm{~ms}$ readout uniform-density spiral design used in section 4 . The proposed variable-density design provided higher spatial resolution and reduced off-resonance artifacts, and thus better spatial localization of flow (Figure 12b). Some aliasing artifacts were observed in spatial domain (see asterisk), but these were not 
observed in the time-velocity distributions. A fully sampled reference is shown in Figure 12c, for comparison.
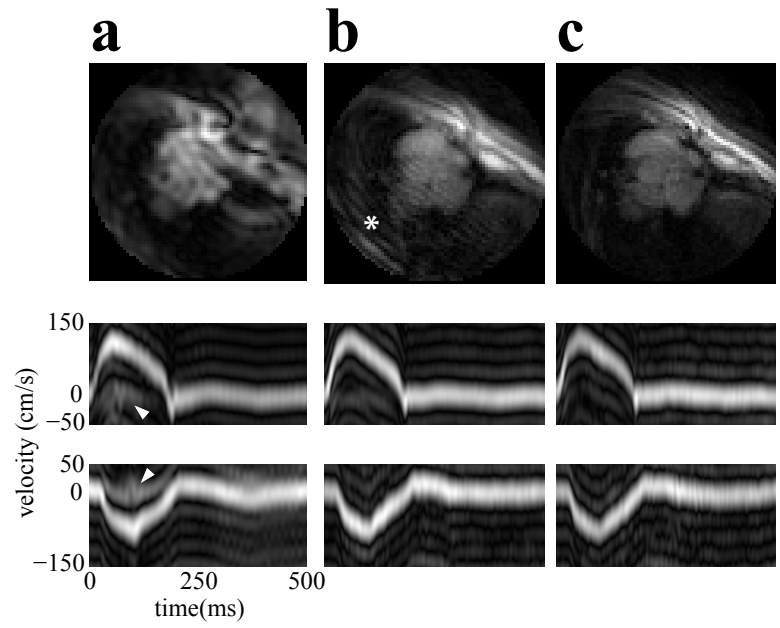

Fig. 12. Effect of variable-density sampling on image quality and spatial localization of flow: (a) original design; (b) new design; (c) ground truth reference. Top row: spatial images from the first cardiac phase; center row: time-velocity distributions measured at the aortic valve; bottom row: time-velocity distributions measured in the descending aorta. The use of higher spatial resolution and shorter readout duration improves the spatial localization of flow, which is identified by the reduced signal from static material in the time-velocity histograms (see arrows). Some aliasing artifacts were observed in spatial domain (see asterisk), but these were not observed in the time-velocity distributions.

Figure 13 contains results from the temporal acceleration experiment. Figure 13a shows the undersampled data in both $v-f$ and $v-t$ domains (compare this with Figure 11). Aliasing components were significantly reduced using the proposed 2D filter (dashed lines), while all of the signal energy was preserved (Figure 13b). The notch filter (dotted line) removed the majority of the remaining aliasing at \pm 20 and $\pm 40 \mathrm{~Hz}$ (solid arrows) (Figure 13c). These results show that the proposed temporal acceleration scheme is capable of achieving 6-fold acceleration in multi-interleaf spiral FVE, without noticeable loss of temporal resolution, and without introducing significant artifacts. A result using view sharing is shown in Figure 13d, for comparison. This approach is equivalent to a moving-average low-pass filter, which reduces the temporal frequency bandwidth (dashed arrows), and causes loss of temporal resolution, perceived as blurring along $t$ (circled).

Figure 14 shows a comparison between the accelerated results and the fully sampled reference. Two-fold acceleration was achieved using variable-density sampling (Figure 14b), with no noticeable artifacts in the time-velocity histogram when compared with the fully sampled reference (Figure 14a). Additional 6-fold acceleration was achieved using the proposed temporal acceleration scheme (Figure 14c). Those results were achieved in a single 18-heartbeat acquisition, while a fully sampled acquisition with the same scan parameters would require 216 heartbeats. Partial Fourier was then used to reduce the acquisition time to 12 heartbeats (i.e., by 1.5 -fold), which represents a combined 18-fold acceleration (Figure 14d). 


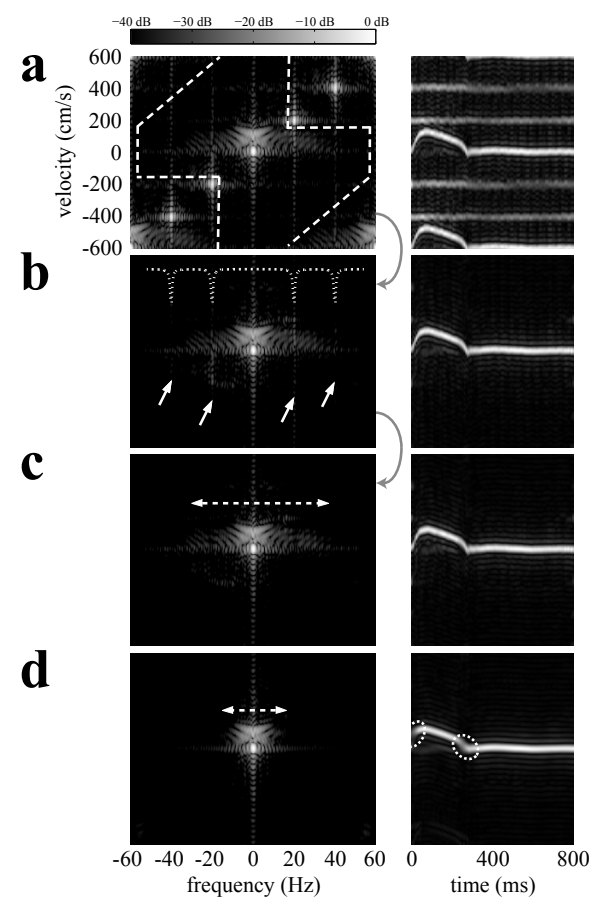

Fig. 13. Temporal acceleration compared with view sharing in (left) $v$ - $f$ space and (right) $v-t$ space: (a) undersampled data; (b) with 2D filtering; (c) with 2D and notch filtering (proposed approach); and (d) with view sharing (conventional approach). The 2D filter (dashed lines) removes a majority of the aliasing, and the notch filter (dotted line) removes the remaining aliasing signal (solid arrows). The proposed method removes aliasing components without noticeable loss of temporal resolution. View sharing reduces the temporal frequency bandwidth (dashed arrows), which causes temporal blurring (circles). Compare the $v-f$ representation in (a) with Figure 11.

No significant artifacts were observed when comparing the reference dataset with the 18 -fold accelerated result.

Figure 15 presents time-velocity distributions from multiple ROIs. These distributions were reconstructed from the 18-fold undersampled dataset used in Figure 14d. Very few artifacts were observed in time-velocity histograms measured in voxels from different locations in the heart. Artifacts could be further reduced by designing different 2D filters for each ROI, based on typical characteristics of the targeted flow. For example, the artifacts observed in the descending aorta (see arrow), could be reduced by more aggressively filtering high positive-velocity components, as no ascending flow is expected in that vessel. These results, when compared with those in Figure 8, also illustrate the different improvements achieved with this approach. The spatial resolution was improved from $7 \mathrm{~mm}$ to $3.6 \mathrm{~mm}$, and off-resonance effects were reduced. The velocity FOV was increased from \pm 400 to $\pm 600 \mathrm{~cm} / \mathrm{s}$, without loss of velocity resolution $(33 \mathrm{~cm} / \mathrm{s})$. The effective temporal resolution was improved from $26 \mathrm{~ms}$ to $9 \mathrm{~ms}$, and ghosting artifacts due to view sharing were eliminated. Both acquisitions were performed in 12-heartbeat breath-holds. 

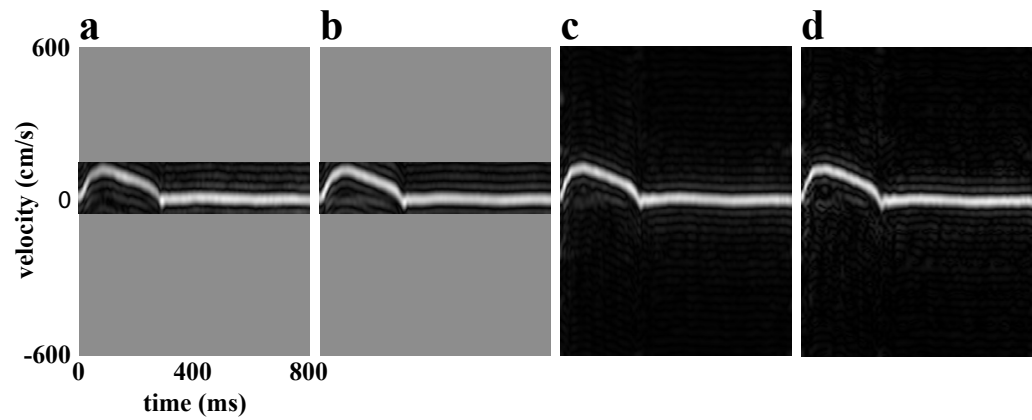

Fig. 14. Accelerated spiral FVE results: (a) fully sampled reference (36 heartbeats); (b) 2-fold acceleration, using variable-density sampling (18 heartbeats); (c) 12-fold acceleration, using variable-density sampling and temporal acceleration (18 heartbeats); and (d) 18-fold acceleration, using variable-density sampling, temporal acceleration, and partial Fourier (12 heartbeats). A reduced velocity FOV (200 cm/s) was used in (a) and (b) to limit the acquisition to a single feasible breath-hold. If acquiring a full $1200 \mathrm{~cm} / \mathrm{s}$ velocity FOV, as in (c) and (d), the total acquisition time for (a) and (b) would have been 216 and 108 heartbeats, respectively. All other scan parameters were identical for the four acquisitions. No significant differences were observed when comparing the 18-fold accelerated result with the fully sampled reference.
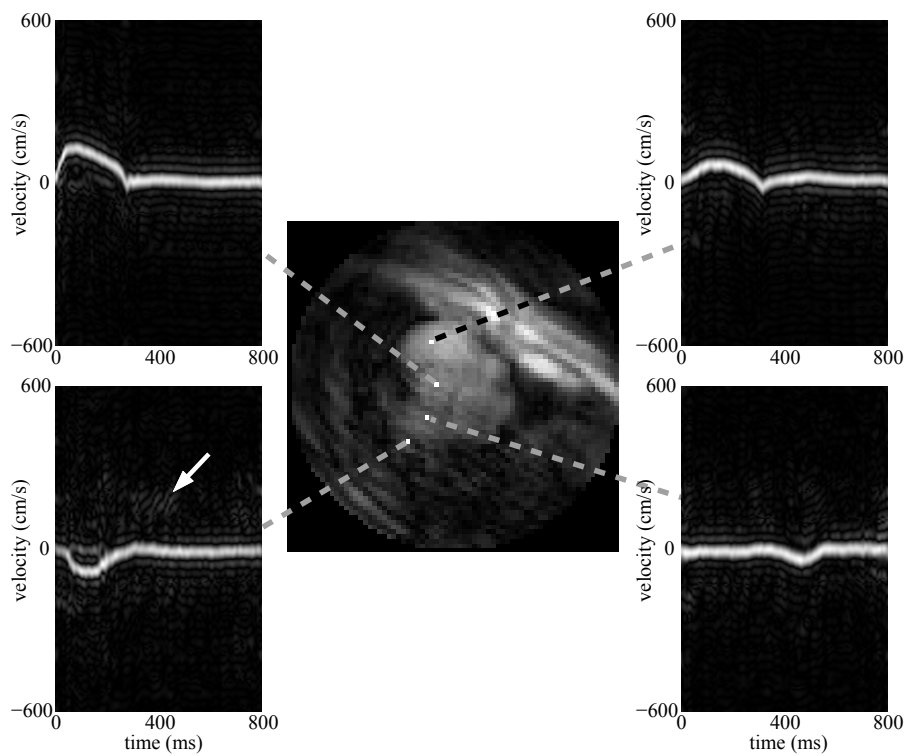

Fig. 15. Flow in multiple ROIs measured in a single 12-heartbeat spiral FVE acquisition using 18-fold acceleration. Voxels of interest are highlighted. Time-velocity histograms reveal only minimal artifacts (see arrow). The data illustrates significant improvements in spatial resolution, velocity field-of-view, and temporal resolution (compare with Figure 8). 
Signal-to-noise ratio was not a limiting issue for the presented application. This is in part due to the large voxel sizes and the multi-dimensional characteristics of spiral FVE. The proposed acceleration scheme reduced scan time and improved spatiotemporal resolution, and did not compromise the quality of the time-velocity histograms. Aliasing artifacts in spatial domain due to variable-density sampling are negligible in time-velocity domain (Figure 12b). The proposed $k$ - $t$ filters remove a majority of the temporal aliasing artifacts, and also filter high-frequency noise for high velocities (Figure 13c). Partial Fourier acceleration may cause some artifacts due to the use of a low-resolution phase estimate, thus a low acceleration factor was used (Figure 14d). Further acceleration could be achieved using parallel imaging techniques (Pruessmann et al., 2001; Samsonov et al., 2006), and further reduction in off-resonance effects could be achieved by imaging at lower field strengths.

\section{Conclusion}

In this chapter, we have addressed the issue of non-invasive aortic valve flow quantitation through magnetic resonance imaging. We addressed both imaging and reconstruction aspects, including accelerated acquisitions and reconstruction from undersampled data. We introduced spiral FVE, a new method for MR flow quantitation, which is capable of accurately capturing peak velocities in flow jets due to stenosis or regurgitation. Spiral FVE compared well against Doppler ultrasound, the current gold standard for cardiovascular flow imaging, and against high-resolution 2DFT phase contrast, the current MRI gold standard. Our method was demonstrated in both healthy volunteers and in a patient.

Using a combination of three different techniques (variable-density spirals, temporal acceleration, and partial Fourier reconstruction), we are able to improve the spiral FVE method by 18 -fold. Improvements consisted of increased velocity field-of-view, higher spatial resolution, reduced off-resonance effects, and higher temporal resolution. The improved acquisition was performed in only 12 heartbeats, whereas 216 heartbeats would be necessary to achieve such improvements without acceleration. No significant artifacts were observed.

Magnetic resonance imaging is potentially the most appropriate technique for addressing all aspects of cardiovascular disease examination. The evaluation of valvular disease and intracardiac flow will be a necessary capability in a comprehensive cardiac MR examination. The imaging and reconstruction techniques proposed in this chapter can be an important contribution towards making such exam feasible.

\section{References}

Ahn, C. B., Park, J. H. \& Cho, Z. H. (1986). High-speed spiral-scan echo planar NMR imaging, IEEE Trans Med Imaging 5(1): 2-7.

Bartels, R. H., Beatty, J. C. \& Barsky, B. A. (1987). An introduction to splines for use in computer graphics and geometric modelling, Morgan Kaufmann, San Francisco, California.

Bernstein, M. A., Fain, S. B. \& Riederer, S. J. (2001). Effect of windowing and zero-filled reconstruction of MRI data on spatial resolution and acquisition strategy, J Magn Reson Imaging 14(3): 270-280.

Bernstein, M. A., King, K. F. \& Zhou, X. J. (2004). Handbook of MRI Pulse Sequences, Academic Press.

Carvalho, J. L. A., DiCarlo, J. C., Kerr, A. B. \& Nayak, K. S. (2007). Reconstruction of variable-density data in Fourier velocity encoding, Proc, ISMRM, 15th Annual Meeting, Berlin, p. 2514. 
Carvalho, J. L. A. \& Nayak, K. S. (2007). Rapid quantitation of cardiovascular flow using slice-selective Fourier velocity encoding with spiral readouts, Magn Reson Med 57(4): 639-646.

Carvalho, J. L. A., Nielsen, J. F. \& Nayak, K. S. (2010). Feasibility of in vivo measurement of carotid wall shear rate using spiral Fourier velocity encoded MRI, Magn Reson Med 63(6): 1537-1547.

Chia, J. M., Fischer, S. E., Wickline, S. A. \& Lorenz, C. H. (2000). Arrhythmia rejection using a VCG-based triggering algorithm, Proc, ISMRM, 8th Annual Meeting, Denver, p. 201.

Clarke, G. D., Hundley, W. G., McColl, R. W., Eckels, R., Smith, D., Chaney, C., Li, H. F. \& Peshock, R. M. (1996). Velocity-encoded, phase-difference cine MRI measurements of coronary artery flow: dependence of flow accuracy on the number of cine frames, J Magn Reson Imaging 6(5): 733-742.

DiCarlo, J. C., Hargreaves, B. A., Nayak, K. S., Hu, B. S., Pauly, J. M. \& Nishimura, D. G. (2005). Variable-density one-shot Fourier velocity encoding, Magn Reson Med 54(3): 645-655.

Dumoulin, C. L., Souza, S. P., Hardy, C. J. \& Ash, S. A. (1991). Quantitative measurement of blood flow using cylindrically localized Fourier velocity encoding, Magn Reson Med 21(2): 242-250.

Feinberg, D. A., Crooks, L. E., Sheldon, P., Hoenninger III, J., Watts, J. \& Arakawa, M. (1985). Magnetic resonance imaging the velocity vector components of fluid flow, Magn Reson Med 2(6): 555-566.

Feinberg, D. A. \& Oshio, K. (1992). Gradient-echo shifting in fast MRI techniques (GRASE imaging) for correction of field inhomogeneity errors and chemical shift, J Magn Reson 97: 177-183.

Galea, D., Lauzon, M. L. \& Drangova, M. (2002). Peak velocity determination using fast Fourier velocity encoding with minimal spatial encoding, Med Phys 29(8): 1719-1728.

Gamper, U., Boesiger, P. \& Kozerke, S. (2008). Compressed sensing in dynamic MRI, Magn Reson Med 59(2): 365-373.

Gao, J. H., Holland, S. K. \& Gore, J. C. (1988). Nuclear magnetic resonance signal from flowing nuclei in rapid imaging using gradient echoes, Med Phys 15(6): 809-814.

Glover, G. H. \& Pelc, N. J. (1988). A rapid-gated cine MRI technique, Magn Reson Annu pp. 299-333.

Hahn, E. L. (1960). Detection of sea-water motion by nuclear precession, J Geophys Res 65(2): 776-777.

Hansen, M. S., Baltes, C., Tsao, J., Kozerke, S., Pruessmann, K. P., Boesiger, P. \& Pedersen, E. M. (2004). Accelerated dynamic Fourier velocity encoding by exploiting velocity-spatio-temporal correlations, MAGMA 17(2): 86-94.

Hargreaves, B. A. (2001). Spin-Manipulation Methods for Efficient Magnetic Resonance Imaging, $\mathrm{PhD}$ thesis, Stanford University.

Hennig, J., Mueri, M., Brunner, P. \& Friedburg, H. (1988). Fast and exact flow measurements with the fast Fourier flow technique, Magn Reson Med 6(4): 369-372.

Holsinger, A. E., Wright, R. C., Riederer, S. J., Farzaneh, F., Grimm, R. C. \& Maier, J. K. (1990). Real-time interactive magnetic resonance imaging, Magn Reson Med 14(3): 547-553.

Hoskins, P. R. (1996). Accuracy of maximum velocity estimates made using Doppler ultrasound systems, Br J Radiol 69(818): 172-177.

Hu, B. S., Pauly, J. M. \& Macovski, A. (1993). Localized real-time velocity spectra determination, Magn Reson Med 30(3): 393-398. 
Irarrazabal, P., Hu, B. S., Pauly, J. M. \& Nishimura, D. G. (1993). Spatially resolved and localized real-time velocity distribution, Magn Reson Med 30(2): 207-212.

Jackson, J. I., Meyer, C. H., Nishimura, D. G. \& Macovski, A. (1991). Selection of a convolution function for Fourier inversion using gridding, IEEE Trans Med Imaging 10(3): 473-478.

Liu, C.-Y., Varadarajan, P., Pohost, G. M. \& Nayak, K. S. (2008). Real-time color-flow MRI at 3 T using variable-density spiral phase contrast, Magn Reson Imaging 26(5): 661-666.

Macgowan, C. K., Kellenberger, C. J., Detsky, J. S., Roman, K. \& Yoo, S. J. (2005). Real-time Fourier velocity encoding: an in vivo evaluation, J Magn Reson Imaging 21(3): 297-304.

Madore, B., Glover, G. H. \& Pelc, N. J. (1999). Unaliasing by Fourier-encoding the overlaps using the temporal dimension (UNFOLD), applied to cardiac imaging and fMRI, Magn Reson Med 42(5): 813-828.

Meyer, C. H., Hu, B. S., Nishimura, D. G. \& Macovski, A. (1992). Fast spiral coronary artery imaging, Magn Reson Med 28(2): 202-213.

Mohiaddin, R. H., Gatehouse, P. D., Henien, M. \& Firmin, D. N. (1997). Cine MR Fourier velocimetry of blood flow through cardiac valves: comparison with Doppler echocardiography., J Magn Reson Imaging 7(4): 657-663.

Moran, P. R. (1982). A flow velocity zeugmatographic interlace for NMR imaging in humans, Magn Reson Imaging 1(4): 197-203.

Moran, P. R., Moran, R. A. \& Karstaedt, N. (1985). Verification and evaluation of internal flow and motion. true magnetic resonance imaging by the phase gradient modulation method, Radiology 154(2): 433-441.

Nayak, K. S., Pauly, J. M., Kerr, A. B., Hu, B. S. \& Nishimura, D. G. (2000). Real-time color flow MRI, Magn Reson Med 43: 251-258.

Nayler, G. L., Firmin, D. N. \& Longmore, D. B. (1986). Blood flow imaging by cine magnetic resonance, J Comput Assist Tomogr 10: 715-722.

Nishimura, D. G. (2010). Principles of Magnetic Resonance Imaging, Stanford University, Palo Alto, California.

Noll, D. C., Meyer, C. H., Pauly, J. M., Nishimura, D. G. \& Macovski, A. (1991). A homogeneity correction method for magnetic resonance imaging with time-varying gradients, IEEE Trans Med Imaging 10(4): 629-637.

Noll, D. C., Nishimura, D. G. \& Macovski, A. (1991). Homodyne detection in magnetic resonance imaging, IEEE Trans Med Imaging 10(2): 154-163.

O'Donnell, M. (1985). NMR blood flow imaging using multiecho, phase contrast sequences, Med Phys 12(1): 59-64.

Pruessmann, K. P., Weiger, M., Bornert, P. \& Boesiger, P. (2001). Advances in sensitivity encoding with arbitrary k-space trajectories, Magn Reson Med 46(4): 638-651.

Rebergen, S. A., van der Wall, E. E., Doornbos, J. \& de Roos, A. (1993). Magnetic resonance measurement of velocity and flow: technique, validation, and cardiovascular applications, Am Heart J 126(6): 1439-1456.

Riederer, S. J., Tasciyan, T., Farzaneh, F., Lee, J. N., Wright, R. C. \& Herfkens, R. J. (1988). MR fluoroscopy: technical feasibility, Magn Reson Med 8(1): 1-15.

Riederer, S. J., Wright, R. C., Ehman, R. L., Rossman, P. J., Holsinger-Bampton, A. E., Hangiangdreu, N. J. \& Grimm, R. C. (1991). Real-time interactive color flow MR imaging, Radiology 181: 33-39.

Samsonov, A. A., Block, W. F., Arunachalam, A. \& Field, A. S. (2006). Advances in locally constrained k-space-based parallel MRI, Magn Reson Med 55(2): 431-438. 
Santos, J. M., Kerr, A. B., Lee, D., McConnell, M. V., Yang, P. C., Hu, B. S. \& Pauly, J. M. (2007). Comprehensive valve evaluation system, Proc, ISMRM, 15th Annual Meeting, Berlin, p. 2551.

Singer, J. R. (1959). Blood flow rates by nuclear magnetic resonance measurements, Science 130(3389): 1652-1653.

Singer, J. R. \& Crooks, L. E. (1983). Nuclear magnetic resonance blood flow measurements in the human brain, Science 221(4611): 654-656.

Tang, C., Blatter, D. D. \& Parker, D. L. (1993). Accuracy of phase-contrast flow measurements in the presence of partial-volume effects, J Magn Reson Imaging 3(3): 377-385.

Tsai, C.-M. \& Nishimura, D. G. (2000). Reduced aliasing artifacts using variable-density k-space sampling trajectories, Magn Reson Med 43: 452-458.

Tsai, C.-M., Olcott, E. W. \& Nishimura, D. G. (1999). Flow quantification using low-spatial-resolution and low-velocity-resolution velocity images, Magn Reson Med 42(4): 682-690.

Tsao, J. (2002). On the UNFOLD method, Magn Reson Med 47(1): 202-207.

van Dijk, P. (1984). Direct cardiac NMR imaging of heart wall and blood flow velocity, J Comput Assist Tomogr 8(3): 429-436.

Winkler, A. J. \& Wu, J. (1995). Correction of intrinsic spectral broadening errors in Doppler peak velocity measurements made with phased sector and linear array transducers, Ultrasound Med Biol 21(8): 1029-1035.

Zur, Y., Wood, M. L. \& Neuringer, L. J. (1991). Spoiling of transverse magnetization in steady-state sequences, Magn Reson Med 21(2): 251-263. 


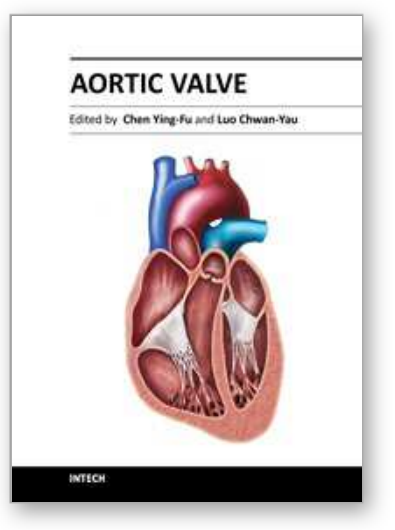

\author{
Aortic Valve \\ Edited by Prof. Chen Ying-Fu
}

ISBN 978-953-307-561-7

Hard cover, 350 pages

Publisher InTech

Published online 09, December, 2011

Published in print edition December, 2011

Much has evolved in the field of aortic valve disease because of the increase in knowledge in the last decade, especially in the area of its management. This book "Aortic Valve" is comprised of 18 chapters covering basic science, general consideration of aortic valve disease, infective endocarditis, aortic sclerosis and aortic stenosis, bioprosthetic valve, transcatheter aortic valve implantation and a special section on congenital anomalies of the aortic valve. We hope this book will be particularly useful to cardiologists and cardiovascular surgeons and trainees. We also believe that this book will be a valuable resource for radiologists, pathologists, cardiovascular anesthesiologists, and other healthcare professionals who have a special interest in treating patients with aortic valve disease. We are certain that information in this book will help to provide virtually most new areas of aortic valve disease that will be employed in the current era.

\title{
How to reference
}

In order to correctly reference this scholarly work, feel free to copy and paste the following:

Joao L. A. Carvalho and Krishna S. Nayak (2011). Rapid Quantitation of Aortic Valve Flow Using Spiral Fourier Velocity Encoded MRI, Aortic Valve, Prof. Chen Ying-Fu (Ed.), ISBN: 978-953-307-561-7, InTech, Available from: http://www.intechopen.com/books/aortic-valve/rapid-quantitation-of-aortic-valve-flow-using-spiral-fouriervelocity-encoded-mri

\section{INTECH}

open science | open minds

\author{
InTech Europe \\ University Campus STeP Ri \\ Slavka Krautzeka 83/A \\ 51000 Rijeka, Croatia \\ Phone: +385 (51) 770447 \\ Fax: +385 (51) 686166 \\ www.intechopen.com
}

\author{
InTech China \\ Unit 405, Office Block, Hotel Equatorial Shanghai \\ No.65, Yan An Road (West), Shanghai, 200040, China \\ 中国上海市延安西路65号上海国际贵都大饭店办公楼 405 单元 \\ Phone: +86-21-62489820 \\ Fax: +86-21-62489821
}


(C) 2011 The Author(s). Licensee IntechOpen. This is an open access article distributed under the terms of the Creative Commons Attribution 3.0 License, which permits unrestricted use, distribution, and reproduction in any medium, provided the original work is properly cited. 